\title{
Cluster Anti-Synchronization of Complex Networks with Nonidentical Dynamical Nodes
}

\author{
Shuguo Wang, ${ }^{1}$ Chunyuan He, ${ }^{1}$ and Hongxing Yao $^{2}$ \\ ${ }^{1}$ Department of Mathematics and Physics, Changzhou Campus, Hohai University, Jiangsu, \\ Changzhou 213022, China \\ ${ }^{2}$ Faculty of Science, Jiangsu University, Zhenjiang, Jiangsu 212013, China
}

Correspondence should be addressed to Shuguo Wang,wsg97@163.com

Received 5 June 2012; Accepted 19 June 2012

Academic Editor: Chong Lin

Copyright (C) 2012 Shuguo Wang et al. This is an open access article distributed under the Creative Commons Attribution License, which permits unrestricted use, distribution, and reproduction in any medium, provided the original work is properly cited.

This paper investigates a new cluster antisynchronization scheme in the time-varying delays coupled complex dynamical networks with nonidentical nodes. Based on the community structure of the networks, the controllers are designed differently between the nodes in one community that have direct connections to the nodes in other communities and the nodes without direct connections with the nodes in other communities strategy; some sufficient criteria are derived to ensure cluster anti-synchronization of the network model. Particularly, the weight configuration matrix is not assumed to be irreducible. The numerical simulations are performed to verify the effectiveness of the theoretical results.

\section{Introduction}

During the past decade, the research on synchronization and dynamical behavior analysis of complex network systems has become a new and important direction in this field [1-6]; many control approaches and many different synchronization phenomena have been developed, such as impulsive control, pinning control and complete synchronization, phase synchronization, cluster synchronization, mixed synchronization, and generalized synchronization, which have been investigated since ten years ago in $[7,8]$ and references therein.

Cluster synchronization means that nodes in the same group synchronize with each other, but there is no synchronization between nodes in different groups $[9,10]$. Wang et al. [11] investigated the cluster synchronization of the dynamical networks with community structure and nonidentical nodes and with identical local dynamics for all individual nodes in each community, which were considered by using some feedback control schemes. Wu and $\mathrm{Lu}$ [12] investigated cluster synchronization in the adaptive complex dynamical networks 
with nonidentical nodes by a local control method and a novel adaptive strategy for the coupling strengths of the networks. Qin and Chen [13] investigated the stability of selected cluster synchronization in coupled Josephson equations. Ma et al. [14] showed that the arbitrarily selected cluster synchronization manifolds could be stabilized by constructing a special coupled matrix for connected chaotic networks. Wu et al. [15] investigated the antisynchronization (AS) problem of two general complex dynamical networks with nondelayed and delayed coupling using pinning adaptive control method. Based on Lyapunov stability theory and Barbalat lemma, a sufficient condition is derived to guarantee the AS between two networks with nondelayed and delayed coupling. However, there is few theoretical result on the cluster anti-synchronization of linearly coupled complex networks with time-varying delays coupling.

Motivated by the aforementioned discussions, this paper aims to analyze the cluster anti-synchronization problem for the time-varying delays coupled complex dynamical networks. The main contributions of this paper are threefold: (1) the local dynamics in each community are identical, but those of different communities are nonidentical. (2) For achieving the synchronization, based on the community structure of the networks, the controllers are designed differently between the nodes in one community which have direct connections to the nodes in other communities and the nodes without direct connections with the nodes in other communities strategy; some sufficient criteria are derived to ensure cluster anti-synchronization of the network model. (3) According to Lyapunov stability theory, the sufficient conditions for achieving cluster anti-synchronization are obtained analytically. Compared with some similar designs, our controllers are very simple.

The paper is organized as follows: the network model is introduced followed by some definitions, lemmas, and hypotheses in Section 2. The cluster anti-synchronization of the complex coupled networks is discussed in Section 3. Simulations are obtained in Section 4. Finally, in Section 5 the various conclusions are discussed.

\section{Model and Preliminaries}

The network with time-varying delays coupling can be described by

$$
c \dot{x}_{i}(t)=f_{\phi_{i}}\left(t, x_{i}(t)\right)+c \sum_{j=1}^{N} a_{i j} \Gamma_{1} x_{j}(t)+c \sum_{j=1}^{N} b_{i j} \Gamma_{2} x_{j}\left(t-\eta_{\phi_{i}}(t)\right), \quad i=1,2, \ldots, N,
$$

where $x_{i}(t)=\left(x_{i 1}(t), x_{i 2}(t), \ldots, x_{i n}(t)\right)^{T} \in R^{n}$ is the state vector of node $i, c>0$ is the coupling strength, and $f_{\phi_{i}}: R^{n} \rightarrow R^{n}$ describes the local dynamics of nodes in the $\phi_{i}$ th community. For any pair of nodes $i$ and $j$, if $\phi_{i} \neq \phi_{j}$, that is, nodes $i$ and $j$ belong to different communities, then $f_{\phi_{i}} \neq f_{\phi_{j}}, \eta_{\phi_{i}}(t)$ is time-varying delay. $\Gamma_{1} \in R^{n \times n}$ and $\Gamma_{2} \in R^{n \times n}$ are inner-coupling matrices, for simplicity; we assume that $\Gamma_{1}, \Gamma_{2}$ are diagonal matrices with positive diagonal elements, $\Gamma_{1}=\operatorname{diag}\left(\rho_{1}, \rho_{2}, \ldots, \rho_{n}\right)$ with $\rho_{i} \geq 0, \Gamma_{2}=\operatorname{diag}\left(\theta_{1}, \theta_{2}, \ldots, \theta_{n}\right)$ with $\theta_{i} \geq 0 . A=\left(a_{i j}\right)_{N \times N}, B=$ $\left(b_{i j}\right)_{N \times N}$ are the weight configuration matrices. If there is a connection from node $i$ to node $j$ $(j \neq i)$, then $a_{i j}=a_{j i}>0, b_{i j}=b_{j i}>0$; otherwise $a_{i j}=a_{j i}=0, b_{i j}=b_{j i}=0$, and the diagonal elements of matrix $A, B$ are defined as

$$
a_{i i}=-\sum_{j=1, j \neq i}^{N} a_{j i}, \quad b_{i i}=-\sum_{j=1, j \neq i}^{N} b_{j i}, \quad i=1,2, \ldots, N .
$$

Particularly, the weight configuration matrices are not assumed to be irreducible. 
When the control inputs $v_{i}(t), u_{i}(t) \in R^{n}(i=1,2, \ldots, N)$ are introduced, the controlled dynamical network with respect to network (2.1) can be written as

$$
\begin{aligned}
\dot{x}_{i}(t)= & f_{\phi_{i}}\left(t, x_{i}(t)\right)+c \sum_{j=1}^{N} a_{i j} \Gamma_{1} x_{j}(t) \\
& +c \sum_{j=1}^{N} b_{i j} \Gamma_{2} x_{j}\left(t-\eta_{\phi_{i}}(t)\right)+u_{i}(t), \quad \phi_{i}(t) \in \bar{J}_{\phi_{i}}, i=1,2, \ldots, N, \\
\dot{x}_{i}(t)= & f_{\phi_{i}}\left(t, x_{i}(t)\right)+c \sum_{j=1}^{N} a_{i j} \Gamma_{1} x_{j}(t) \\
& +c \sum_{j=1}^{N} b_{i j} \Gamma_{2} x_{j}\left(t-\eta_{\phi_{i}}(t)\right)+v_{i}(t), \quad \phi_{i}(t) \in J_{\phi_{i}}-\bar{J}_{\phi_{i}}, i=1,2, \ldots, N,
\end{aligned}
$$

where $J_{\phi_{i}}$ denotes all the nodes in the $\phi_{i}$ th community and $\bar{J}_{\phi_{i}}$ represents the nodes in the $\phi_{i}$ th community, which have direct links with the nodes in other communities.

In this paper, let $\left\{C_{1}, C_{2}, \ldots, C_{m}\right\}$ denote $m(2 \leq m \leq N)$ communities of the networks and $\bigcup_{i=1}^{m} C_{i}=\{1,2, \ldots N\}$. If node $i$ belongs to the $j$ th community, then we denote $\phi_{i}=j$. We employ $f_{i}(\cdot)$ to represent the local dynamics of all nodes in the $i$ th community. The local dynamics of individual nodes in different communities are assumed to be nonidentical, that is, if $\phi_{i} \neq \phi_{j}$, then $f_{\phi_{i}} \neq f_{\phi_{j}}$. Let $s_{\phi_{i}}(t)$ be a solution of an isolated node in the $\phi_{i}$ th community, that is, $\dot{s}_{i}(t)=f_{\phi_{i}}\left(t, s_{i}(t)\right)$, where $\lim _{t \rightarrow \infty}\left\|s_{i}(t)-s_{j}(t)\right\| \neq 0(i \neq j)$ and the set $S=$ $\left\{s_{1}(t), s_{2}(t), \ldots, s_{m}(t)\right\}$ is used as the cluster anti-synchronization manifold for network (2.3). Cluster anti-synchronization can be realized if and only if the manifold $S$ is stable, where $s_{k}(t)$ may be an equilibrium point, a periodic orbit, or even a chaotic orbit.

Definition 2.1 (see [15]). The dynamical network (2.1) is said to achieve cluster anti-synchronization (CAS) if

$$
c \lim _{t \rightarrow \infty}\left\|e_{i}(t)\right\|=\lim _{t \rightarrow \infty}\left\|x_{i}(t)+s_{\phi_{i}}(t)\right\|=0, \quad i=1,2, \ldots, N
$$

Lemma 2.2. For any two vectors $x$ and $y$, a matrix $Q>0$ with compatible dimensions, one has

$$
2 x^{T} y \leq x^{T} Q x+y^{T} Q^{-1} y .
$$

Assumption 2.3 (see [15]). For any $x=\left(x_{1}, x_{2}, \ldots, x_{n}\right)^{T} \in R^{n}, y=\left(y_{1}, y_{2}, \ldots, y_{n}\right)^{T} \in R^{n}$, there exists a positive constant $L$ such that, $(y-x)^{T}(f(t, y)-f(t, x)) \leq L(y-x)^{T} \Gamma(y-x)$, where $\Gamma$ is a positive definite matrix. Here $x$ and $y$ are time-varying vectors.

Assumption 2.4. $f(x, t)$ is an odd function of $x$, that is, $f(-x, t)=-f(x, t)$ for arbitrary $x \in R^{n}$.

Assumption 2.5. $\eta_{\phi_{i}}(t)$ is a differential function with $0 \leq \dot{\eta}_{\phi_{i}}(t) \leq \varepsilon \leq 1$. Clearly, this assumption is certainly ensured if the delay $\eta_{\phi_{i}}(t)$ is constant. 


\section{Main Results}

In this section, a control scheme is developed to synchronize a delayed complex network with nonidentical nodes to any smooth dynamics $s_{\phi_{i}}(t)$. Let synchronization errors $e_{i}(t)=$ $x_{i}(t)+s_{\phi_{i}}(t)$ for $i=1,2, \ldots, N$, according to system (2.1); the error dynamical system can be derived as

$$
\begin{aligned}
\dot{e}_{i}(t)= & \tilde{f}_{\phi_{i}}\left(t, x_{i}(t)\right)+c \sum_{j=1}^{N} a_{i j} \Gamma_{1} e_{j}(t)+c \sum_{j=1}^{N} b_{i j} \Gamma_{2} e_{j}\left(t-\eta_{\phi_{i}}(t)\right)-c \sum_{i=1}^{N} a_{i j} \Gamma_{1} s_{\phi_{i}}(t) \\
& -c \sum_{i=1}^{N} b_{i j} \Gamma_{2} s_{\phi_{i}}\left(t-\eta_{\phi_{i}}(t)\right)+u_{i}(t), \quad \phi_{i}(t) \in \bar{J}_{\phi_{i}}, i=1,2, \ldots, N, \\
\dot{e}_{i}(t)= & \tilde{f}_{\phi_{i}}\left(t, x_{i}(t)\right)+c \sum_{j=1}^{N} a_{i j} \Gamma_{1} e_{j}(t) \\
& +c \sum_{j=1}^{N} b_{i j} \Gamma_{2} e_{j}\left(t-\eta_{\phi_{i}}(t)\right)+v_{i}(t), \quad \phi_{i}(t) \in J_{\phi_{i}}-\bar{J}_{\phi_{i}}, i=1,2, \ldots, N,
\end{aligned}
$$

where $\tilde{f}_{\phi_{i}}\left(t, e_{i}(t)\right)=f_{\phi_{i}}\left(t, x_{i}(t)\right)+f_{\phi_{i}}\left(t, s_{\phi_{i}}(t)\right)$, for $i=1,2, \ldots, N$.

According to the diffusive coupling condition (2.2) of the matrix $A, B$, we have

$$
c \sum_{i=1}^{N} a_{i j} \Gamma_{1} s_{\phi_{i}}(t)+c \sum_{i=1}^{N} b_{i j} \Gamma_{2} s_{\phi_{i}}\left(t-\eta_{\phi_{i}}(t)\right)=0, \quad i \in J_{\phi_{i}}-\bar{J}_{\phi_{i}} .
$$

On the basis of this property, for achieving cluster anti-synchronization, we design the controllers as follows:

$$
\begin{gathered}
u_{i}(t)=c \sum_{i=1}^{N} a_{i j} \Gamma_{1} s_{\phi_{i}}(t)+c \sum_{i=1}^{N} b_{i j} \Gamma_{2} s_{\phi_{i}}\left(t-\eta_{\phi_{i}}(t)\right)-d_{i} e_{i}(t), \quad i \in \bar{J}_{\phi_{i}}, \\
v_{i}(t)=-d_{i} e_{i}(t), \quad i \in J_{\phi_{i}}-\bar{J}_{\phi_{i}},
\end{gathered}
$$

where $\dot{d}_{i}=k_{i} e_{i}^{T}(t) e_{i}(t), d_{i}$ are the feedback strength and $k_{i}$ are arbitrary positive constants.

It is easy to see that the synchronization of the controlled complex network (2.1) is achieved if the zero solution of the error system (3.1) is globally asymptotically stable, which is ensured by the following theorem. ing result.

Let $\tilde{e}_{j}(t)=\left(e_{1 j}(t), e_{2 j}(t), \ldots, e_{N j}(t)\right)^{T}, D=\operatorname{diag}\left(d_{1}, \ldots, d_{N}\right)^{T}$; then we have the follow-

Theorem 3.1. Suppose Assumptions 2.3-2.5 hold. Consider the network (2.1) via control law (3.3). If the following conditions hold:

$$
\lambda_{\max }(\Gamma) L+c \lambda_{\max }(Q)+\frac{1}{2} c \lambda_{\max }\left(P P^{T}\right)+\frac{c}{2(1-\varepsilon)}<d,
$$


Journal of Applied Mathematics

then the system (2.3) is cluster anti-synchronization, where d is sufficiently large positive constant to be determined.

Proof. From Assumptions 2.3 and 2.4, we get

$$
(y+x)^{T}(f(t, y)+f(t, x))=(y-(-x))^{T}(f(t, y)-f(t,-x)) \leq L(y+x)^{T} \Gamma(y+x)
$$

for $x, y \in R^{n}$.

Construct the following Lyapunov functional:

$$
V(t)=\frac{1}{2} \sum_{i=1}^{N} e_{i}^{T}(t) e_{i}(t)+\frac{c}{2(1-\varepsilon)} \sum_{i=1}^{N} \int_{t-\eta_{\phi_{i}}(t)}^{t} e_{i}^{T}(\xi) e_{i}(\xi) d \xi+\frac{1}{2} \sum_{i=1}^{N} \frac{\left(d_{i}-d\right)^{2}}{k_{i}} .
$$

Calculating the derivative of $V(t)$, we have

$$
\begin{aligned}
\dot{V}(t)= & \sum_{i=1}^{N} e_{i}^{T}(t)\left[\tilde{f}_{\phi_{i}}\left(t, e_{i}(t)\right)+c \sum_{j=1}^{N} a_{i j} \Gamma_{1} e_{j}(t)+c \sum_{j=1}^{N} b_{i j} \Gamma_{2} e_{j}\left(t-\eta_{\phi_{i}}(t)\right)-d_{i} e_{i}(t)\right] \\
& +\frac{c}{2(1-\varepsilon)} \sum_{i=1}^{N} e_{i}^{T}(t) e_{i}(t)-c\left(\frac{1-\dot{\eta}_{\phi_{i}}(t)}{2(1-\varepsilon)}\right) \sum_{i=1}^{N} e_{i}^{T}\left(t-\eta_{\phi_{i}}(t)\right) e_{i}\left(t-\eta_{\phi_{i}}(t)\right) \\
& +\sum_{i=1}^{N}\left(d_{i}-d\right) e_{i}^{T}(t) e_{i}(t) .
\end{aligned}
$$

By the Assumptions 2.3-2.5, we have

$$
\begin{aligned}
\dot{V}(t) \leq & \sum_{i=1}^{N} L e_{i}^{T}(t) \Gamma e_{i}(t)+c \sum_{i=1}^{N} \sum_{j=1}^{N} a_{i j} e_{i}^{T}(t) \Gamma_{1} e_{j}(t)+c \sum_{i=1}^{N} \sum_{j=1}^{N} b_{i j} e_{i}^{T}(t) \Gamma_{2} e_{j}\left(t-\eta_{\phi_{i}}(t)\right) \\
& +\frac{c}{2(1-\varepsilon)} \sum_{i=1}^{N} e_{i}^{T}(t) e_{i}(t)-\frac{c}{2} \sum_{i=1}^{N} e_{i}^{T}\left(t-\eta_{\phi_{i}}(t)\right) e_{i}\left(t-\eta_{\phi_{i}}(t)\right)-\sum_{i=1}^{N} d e_{i}^{T}(t) e_{i}(t) \\
\leq & \sum_{i=1}^{N} L e_{i}^{T}(t) \Gamma e_{i}(t)+c e^{T}\left(A \otimes \Gamma_{1}\right) e+c e^{T}\left(B \otimes \Gamma_{2}\right) e\left(t-\eta_{\phi_{i}}(t)\right)+\frac{c}{2(1-\varepsilon)} \sum_{i=1}^{N} e_{i}^{T}(t) e_{i}(t) \\
& -\frac{c}{2} e^{T}\left(t-\eta_{\phi_{i}}(t)\right) e\left(t-\eta_{\phi_{i}}(t)\right)-d e^{T}(t) e(t) .
\end{aligned}
$$

Let $e(t)=\left(e_{1}^{T}(t), e_{2}^{T}(t), \ldots, e_{N}^{T}(t)\right)^{T} \in R^{n N}, Q=\left(A \otimes \Gamma_{1}\right), P=\left(B \otimes \Gamma_{2}\right)$, where $\otimes$ represents the Kronecker product. Then

$$
\begin{aligned}
\dot{V}(t) \leq & \lambda_{\max }(\Gamma) L e^{T}(t) e(t)+c e^{T}(t) Q e(t)+c e^{T}(t) P e\left(t-\eta_{\phi_{i}}(t)\right)+\frac{c}{2(1-\varepsilon)} e^{T}(t) e(t) \\
& -\frac{c}{2} e^{T}\left(t-\eta_{\phi_{i}}(t)\right) e\left(t-\eta_{\phi_{i}}(t)\right)-d e^{T}(t) e(t) .
\end{aligned}
$$




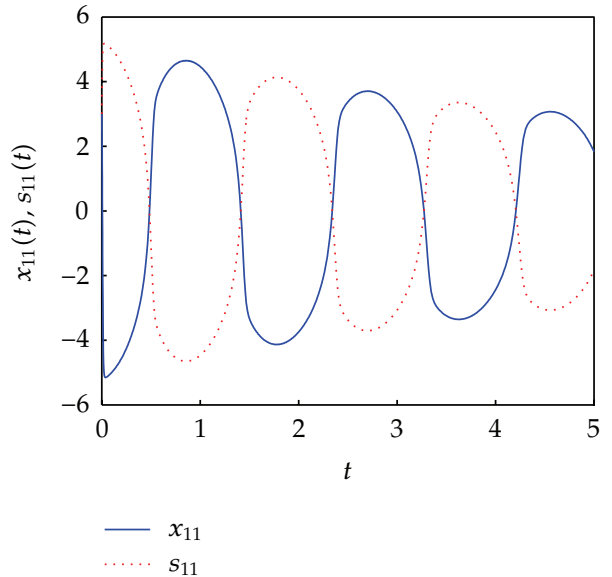

(a)
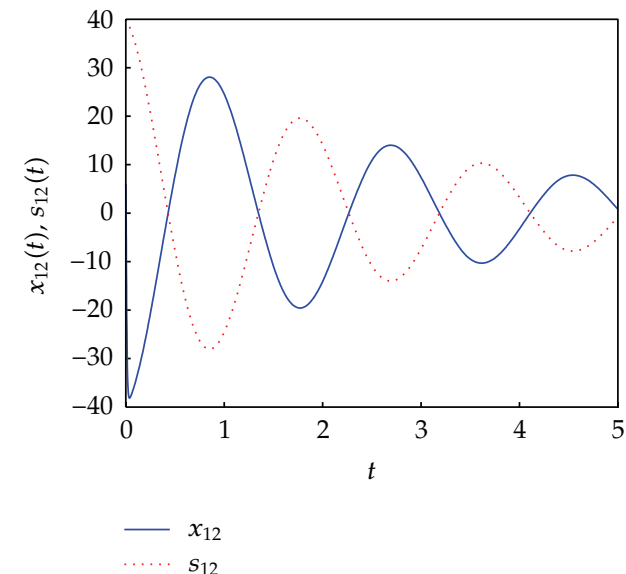

(b)

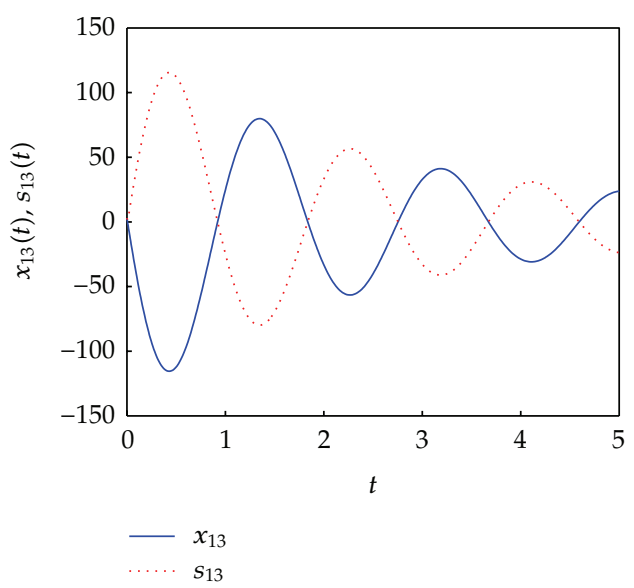

(c)

Figure 1: The trajectories of nodes in the first community.

By Lemma 2.2, we have

$$
\begin{aligned}
\dot{V}(t) & \leq \lambda_{\max }(\Gamma) L e^{T}(t) e(t)+c e^{T}(t) Q e(t)+\frac{c}{2} e^{T}(t) P P^{T} e(t)+\frac{c}{2(1-\varepsilon)} e^{T}(t) e(t)-d e^{T}(t) e(t) \\
& \leq\left(\lambda_{\max }(\Gamma) L+c \lambda_{\max }(Q)+\frac{1}{2} c \lambda_{\max }\left(P P^{T}\right)+\frac{c}{2(1-\varepsilon)}-d\right) e^{T}(t) e(t) .
\end{aligned}
$$

Therefore, if we have $\lambda_{\max }(\Gamma) L+c \lambda_{\max }(Q)+(1 / 2) c \lambda_{\max }\left(P P^{T}\right)+c / 2(1-\varepsilon)<d$, then

$$
\dot{V}(t) \leq-e^{T}(t) e(t) .
$$

Choose $\lambda_{\max }(\Gamma) L+c \lambda_{\max }(Q)+(1 / 2) c \lambda_{\max }\left(P P^{T}\right)+c / 2(1-\varepsilon)+1<d$. Thus, one obtains $\dot{V}(t) \leq-e^{T}(t) e(t) \leq 0$. It is obvious that the largest invariant set contained in set $E=\{\dot{V}(t)=0\}=\left\{e_{i}(t)=0, i=1,2, \ldots, N\right\}$ is $\widetilde{Q}=\left\{e_{i}(t)=0, d_{i}(t)=d, i=1,2, \ldots, N\right\}$. 


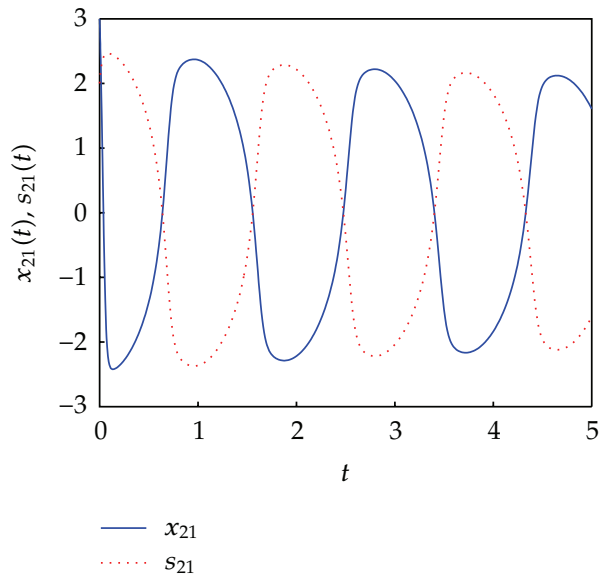

(a)

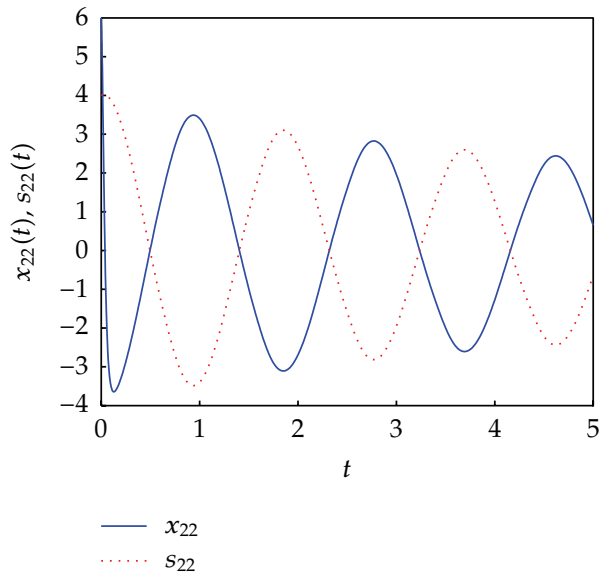

(b)

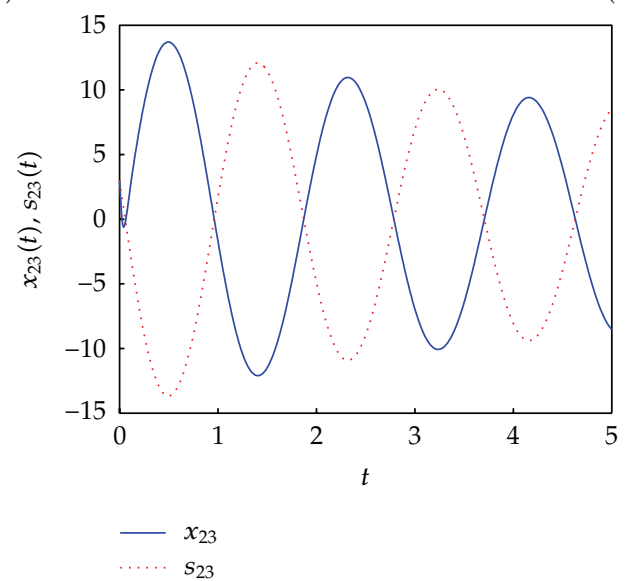

(c)

Figure 2: The trajectories of nodes in the second community.

Based on LaSalle invariance principle, starting with any initial values of the error dynamical system, the trajectory asymptotically converges to the largest invariant $\widetilde{Q}$ which implies that $\lim _{t \rightarrow \infty}\left\|e_{i}(t)\right\|=0$ for $i=1,2, \ldots, N$. Therefore, cluster anti-synchronization in the network (2.3) is achieved under the controllers (3.3). This completes the proof.

Corollary 3.2. When $A=0$, network (2.1) is translated into

$$
\dot{x}_{i}(t)=f_{\phi_{i}}\left(t, x_{i}(t)\right)+c \sum_{j=1}^{N} b_{i j} \Gamma_{2} x_{j}\left(t-\eta_{\phi_{i}}(t)\right), \quad i=1,2, \ldots, N .
$$

We design the controllers, as follows, then the complex networks can also achieve synchronization, where

$$
\begin{gathered}
u_{i}(t)=c \sum_{i=1}^{N} b_{i j} \Gamma_{2} s_{\phi_{i}}\left(t-\eta_{\phi_{i}}(t)\right)-d_{i} e_{i}(t), \quad i \in \bar{J}_{\phi_{i}}, \\
v_{i}(t)=-d_{i} e_{i}(t), \quad i \in J_{\phi_{i}}-\bar{J}_{\phi_{i}} .
\end{gathered}
$$




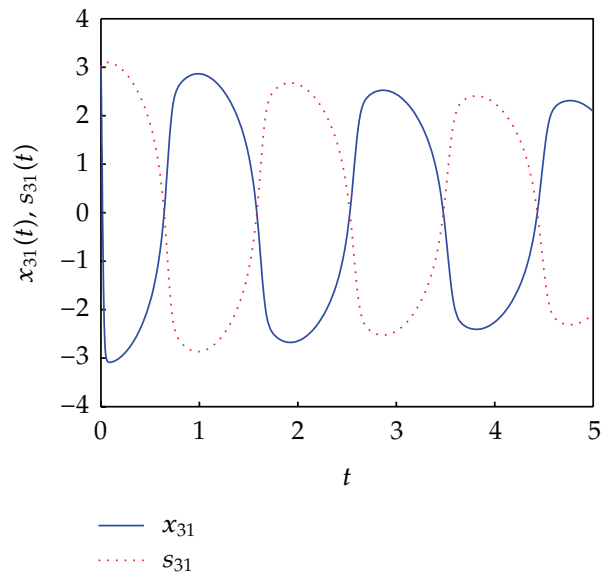

(a)

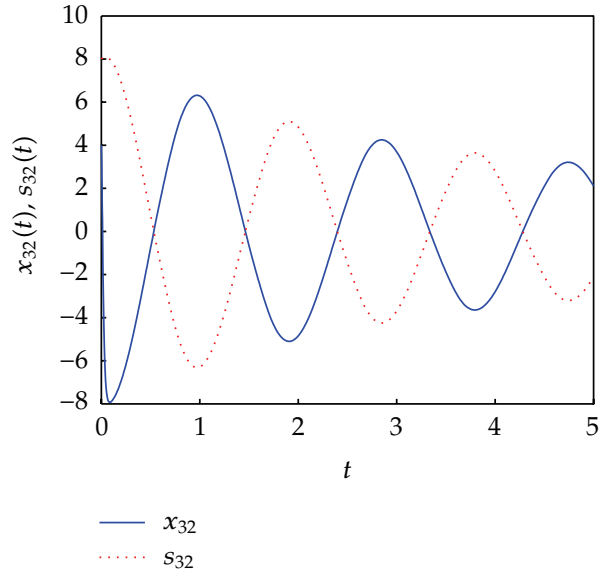

(b)

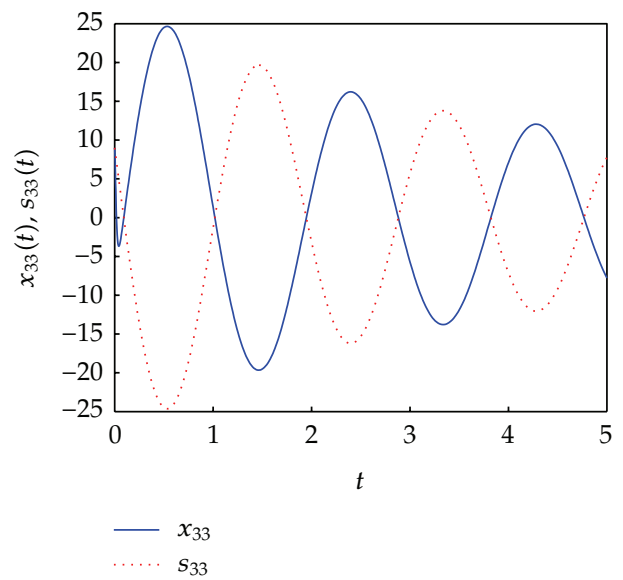

(c)

Figure 3: The trajectories of nodes in the third community.

Corollary 3.3. When $B=0$, network (2.1) is translated into

$$
\dot{x}_{i}(t)=f_{\phi_{i}}\left(t, x_{i}(t)\right)+c \sum_{j=1}^{N} a_{i j} \Gamma_{1} x_{j}(t), \quad i=1,2, \ldots, N
$$

We design the controllers, as follows, then the complex networks can also achieve synchronization, where

$$
\begin{gathered}
u_{i}(t)=c \sum_{i=1}^{N} a_{i j} \Gamma_{1} s_{\phi_{i}}(t)-d_{i} e_{i}(t), \quad i \in \bar{J}_{\phi_{i}} \\
v_{i}(t)=-d_{i} e_{i}(t), \quad i \in J_{\phi_{i}}-\bar{J}_{\phi_{i}} .
\end{gathered}
$$




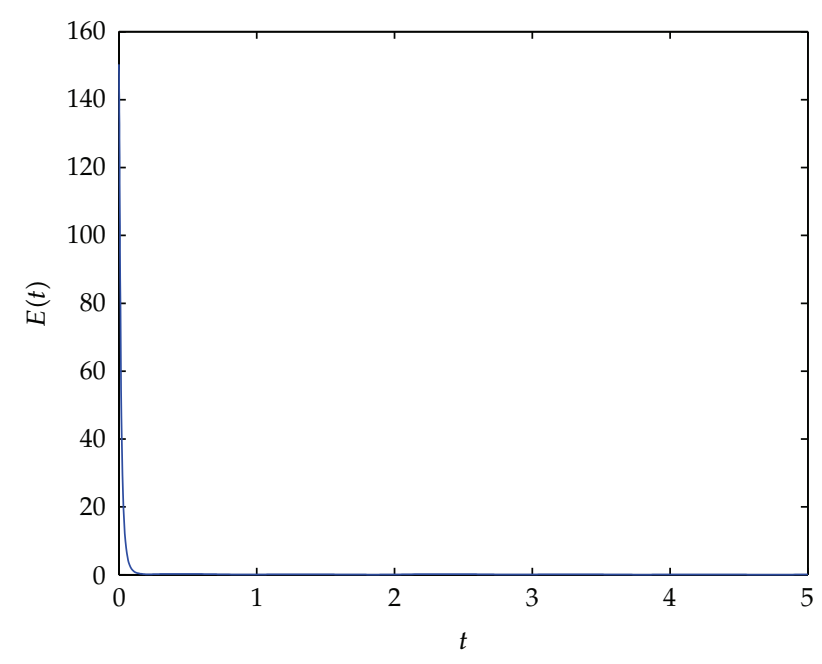

Figure 4: Time evolution of the synchronization error $E(t)$.

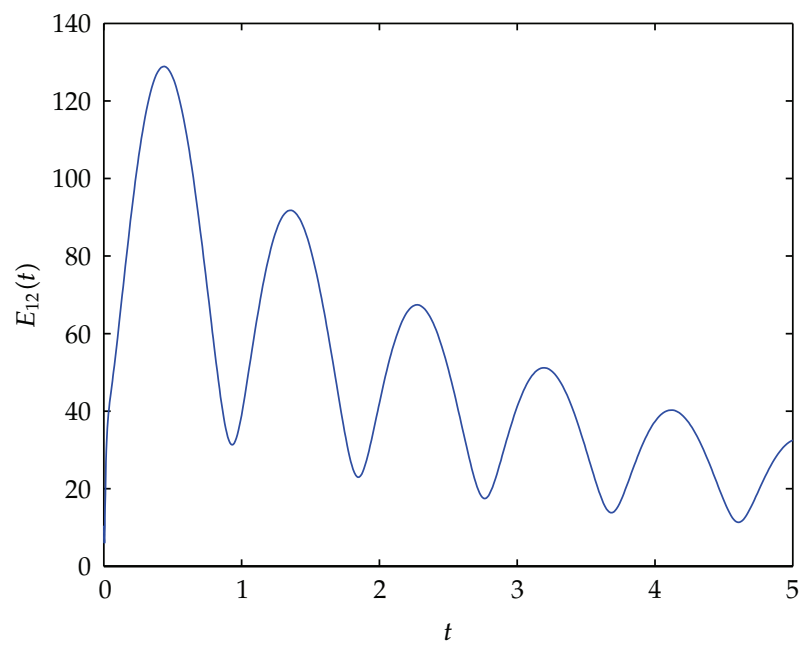

Figure 5: Time evolution of the synchronization error $E_{12}(t)$.

\section{Illustrative Examples}

In this section, several numerical examples are provided to illustrate the proposed synchronization methods. The nodes dynamics are the following well-known modified Chua's circuit [16] with different system parameters. Considering the following network:

$$
\begin{aligned}
\dot{x}_{i}(t)= & f_{\phi_{i}}\left(t, x_{i}(t)\right)+c \sum_{j=1}^{N} a_{i j} \Gamma_{1} x_{j}(t) \\
& +c \sum_{j=1}^{N} b_{i j} \Gamma_{2} x_{j}\left(t-\eta_{\phi_{i}}(t)\right)+u_{i}(t), \quad \phi_{i}(t) \in \bar{J}_{\phi_{i}}, i=1,2, \ldots, N,
\end{aligned}
$$




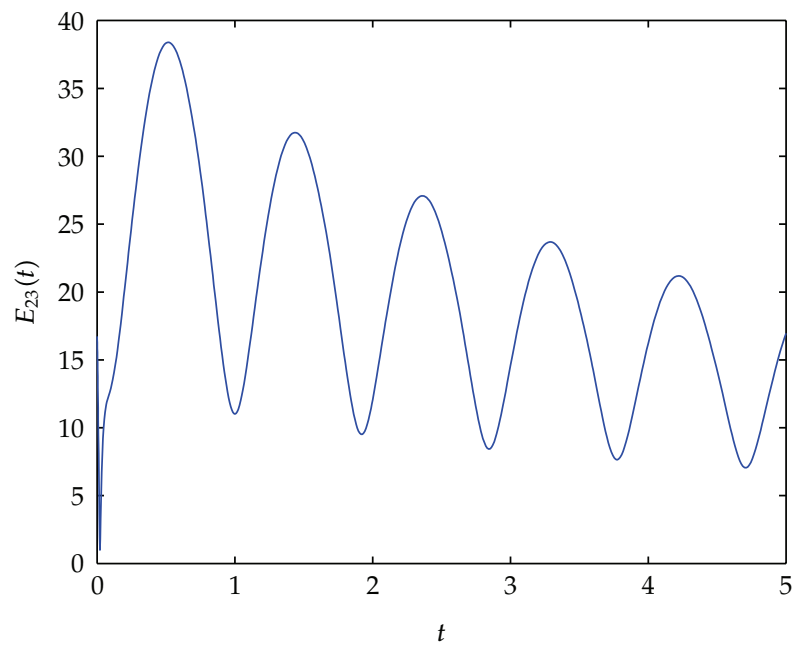

Figure 6: Time evolution of the synchronization error $E_{23}(t)$.

$$
\begin{aligned}
\dot{x}_{i}(t)= & f_{\phi_{i}}\left(t, x_{i}(t)\right)+c \sum_{j=1}^{N} a_{i j} \Gamma_{1} x_{j}(t) \\
& +c \sum_{j=1}^{N} b_{i j} \Gamma_{2} x_{j}\left(t-\eta_{\phi_{i}}(t)\right)+v_{i}(t), \quad \phi_{i}(t) \in J_{\phi_{i}}-\bar{J}_{\phi_{i}}, i=1,2, \ldots, N,
\end{aligned}
$$

where $x_{i}(t)=\left(x_{1 i}(t), x_{2 i}(t), x_{3 i}(t)\right)^{T}, f_{1}\left(t, x_{i}(t)\right)=D_{1} x_{i}(t)+h_{1}\left(x_{i}(t)\right), f_{2}\left(t, x_{i}(t)\right)=D_{2} x_{i}(t)+$ $h_{2}\left(x_{i}(t)\right), f_{3}\left(t, x_{i}(t)\right)=D_{3} x_{i}(t)+h_{3}\left(x_{i}(t)\right), h_{1}\left(x_{i}\right)=h_{2}\left(x_{i}\right)=h_{3}\left(x_{i}\right)=\left((-20 / 7) x_{i 1}^{3}, 0,0\right)^{T}, \Gamma_{1}=$ $\Gamma_{2}=\operatorname{Diag}\{1,1,1\}$,

$$
\begin{gathered}
\eta_{1}(t)=\frac{0.1 e^{t}}{1+e^{t}}, \quad \eta_{2}(t)=\frac{2 e^{t}}{1+e^{t}}, \quad \eta_{3}(t)=\frac{0.8 e^{t}}{1+e^{t}} \\
D_{1}=\left[\begin{array}{ccc}
-\frac{10}{7} & 10 & 0 \\
1 & -1 & 1 \\
0 & -12 & 0
\end{array}\right], \quad D_{2}=\left[\begin{array}{ccc}
-\frac{10}{7} & 10 & 0 \\
1 & -1 & 1 \\
0 & -13 & 0
\end{array}\right], \quad D_{3}=\left[\begin{array}{cccc}
-\frac{10}{7} & 10 & 0 \\
1 & -1 & 1 \\
0 & -14 & 0
\end{array}\right], \\
c=0.01, \quad d=10, \quad A=B=\left[\begin{array}{cccccc}
-2 & 1 & 1 & -2 & 1 & 1 \\
-1 & -3 & 0 & 1 & 2 & 1 \\
1 & 0 & -3 & 1 & 1 & 0 \\
1 & 1 & 1 & -4 & 1 & 0 \\
1 & 1 & 1 & 0 & -4 & 1 \\
1 & 1 & 0 & 0 & 0 & -2
\end{array}\right]
\end{gathered}
$$

Simulation results are given in Figures 1,2, and 3. Cluster anti-synchronization is achieved by the controller (3.3). 


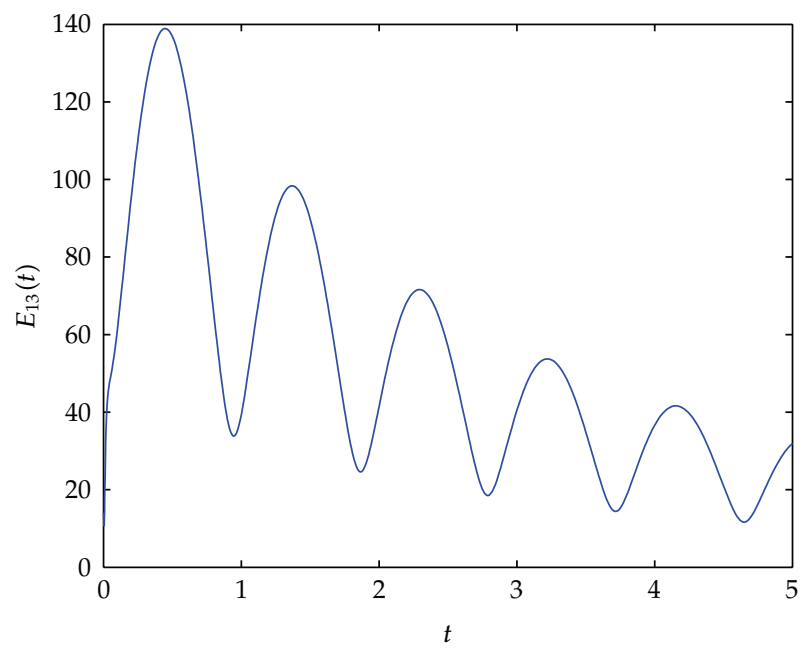

Figure 7: Time evolution of the synchronization error $E_{13}(t)$.

The following quantities are utilized to measure the process of cluster anti-synchronization

$$
\begin{gathered}
E(t)=\sum_{i=1}^{N}\left\|x_{i}(t)+s_{\phi_{i}}(t)\right\|, \\
E_{12}(t)=\left\|x_{u}(t)-x_{v}(t)\right\|, \quad u \in C_{1}, v \in C_{2}, \\
E_{13}(t)=\left\|x_{u}(t)-x_{v}(t)\right\|, \quad u \in C_{1}, v \in C_{3}, \\
E_{23}(t)=\left\|x_{u}(t)-x_{v}(t)\right\|, \quad u \in C_{2}, v \in C_{3},
\end{gathered}
$$

where $E(t)$ is the error of cluster synchronization for this controlled network (2.2); $E_{12}(t)$, $E_{13}(t)$, and $E_{23}(t)$ are the errors between two communities; cluster anti-synchronization is achieved if the synchronization error $E(t)$ converges to zero and $E_{12}(t), E_{13}(t)$, and $E_{23}(t)$ do not as $t \rightarrow \infty$. Simulation results are given in Figures $4,5,6$, and 7 .

\section{Conclusions}

The cluster anti-synchronization in community networks has been studied in this paper, based on the community structure of the networks. Particularly, weight configuration matrix is not assumed to be irreducible. Some simple and useful criteria are derived by constructing an effective control scheme. The synchronization criteria are independent of time delay. Finally, the developed techniques are applied in three complex community networks. The numerical simulations are performed to verify the effectiveness of the theoretical results.

\section{Acknowledgments}

Research is partially supported by the National Nature Science Foundation of China (no. 70871056) and by the Six Talents Peak Foundation of Jiangsu Province. 


\section{References}

[1] L. M. Pecora and T. L. Carroll, "Master stability functions for synchronized coupled systems," Physical Review Letters, vol. 80, no. 10, pp. 2109-2112, 1998.

[2] K. S. Fink, G. Johnson, T. Carroll, D. Mar, and L. Pecora, “Three coupled oscillators as a universal probe of synchronization stability in coupled oscillator arrays," Physical Review E, vol. 61, no. 5, pp. 5080-5090, 2000.

[3] C. Y. Yin, W. X. Wang, G. Chen, and B. H. Wang, "Decoupling process for better synchronizability on scale-free networks," Physical Review E, vol. 74, no. 4, Article ID 047102, 4 pages, 2006.

[4] H. Hong, B. J. Kim, M. Y. Choi, and H. Park, "Factors that predict better synchronizability on complex networks," Physical Review E, vol. 69, no. 6, Article ID 067105, 4 pages, 2004.

[5] D. U. Hwang, M. Chavez, A. Amann, and S. Boccaletti, "Synchronization in complex networks with age ordering," Physical Review Letters, vol. 94, no. 13, Article ID 138701, 4 pages, 2005.

[6] V. Colizza, J. R. Banavar, A. Maritan, and A. Rinaldo, "Network structures from selection principles," Physical Review Letters, vol. 92, no. 19, Article ID 198701, 4 pages, 2004.

[7] T. Ichinomiya, "Frequency synchronization in a random oscillator network," Physical Review E, vol. 70, no. 2, Article ID 026116, 5 pages, 2004.

[8] V. Afraimovich, A. Cordonet, and N. F. Rulkov, "Generalized synchronization of chaos in noninvertible maps," Physical Review E, vol. 66, no. 1, Article ID 016208, 6 pages, 2002.

[9] M. I. Rabinovich, J. J. Torres, P. Varona, R. Huerta, and P. Weidman, “Origin of coherent structures in a discrete chaotic medium," Physical Review E, vol. 60, no. 2, pp. R1130-R1133, 1999.

[10] D. H. Zanette and A. S. Mikhailov, "Mutual synchronization in ensembles of globally coupled neural networks," Physical Review E, vol. 58, no. 1, pp. 872-875, 1998.

[11] K. Wang, X. Fu, and K. Li, "Cluster synchronization in community networks with nonidentical nodes," Chaos, vol. 19, no. 2, Article ID 023106, 10 pages, 2009.

[12] X. J. Wu and H. T. Lu, "Cluster synchronization in the adaptive complex dynamical networks via a novel approach," Physics Letters A, vol. 375, no. 14, pp. 1559-1565, 2011.

[13] W. X. Qin and G. Chen, "Coupling schemes for cluster synchronization in coupled Josephson equations," Physica D, vol. 197, no. 3-4, pp. 375-391, 2004.

[14] Z. Ma, Z. Liu, and G. Zhang, "A new method to realize cluster synchronization in connected chaotic networks," Chaos, vol. 16, no. 2, Article ID 023103, 9 pages, 2006.

[15] Y. Q. Wu, C. P. Li, A. L. Yang, L. J. Song, and Y. J. Wu, "Pinning adaptive anti-synchronization between two general complex dynamical networks with non-delayed and delayed coupling," Applied Mathematics and Computation, vol. 218, no. 14, pp. 7445-7452, 2012.

[16] J. W. Wang, Q. Ma, L. Zeng, and M. S. Abd-Elouahab, "Mixed outer synchronization of coupled complex networks with time-varying coupling delay," Chaos, vol. 21, no. 1, Article ID 013121, 8 pages, 2011. 


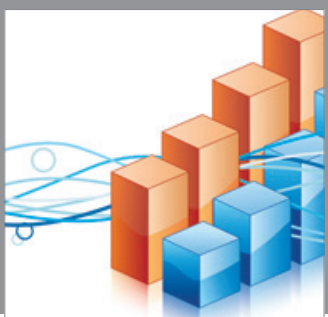

Advances in

Operations Research

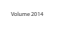

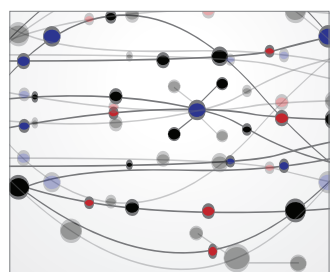

\section{The Scientific} World Journal
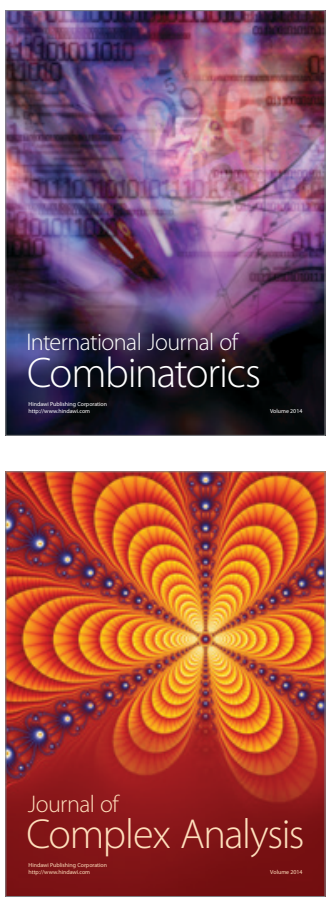

International Journal of

Mathematics and

Mathematical

Sciences
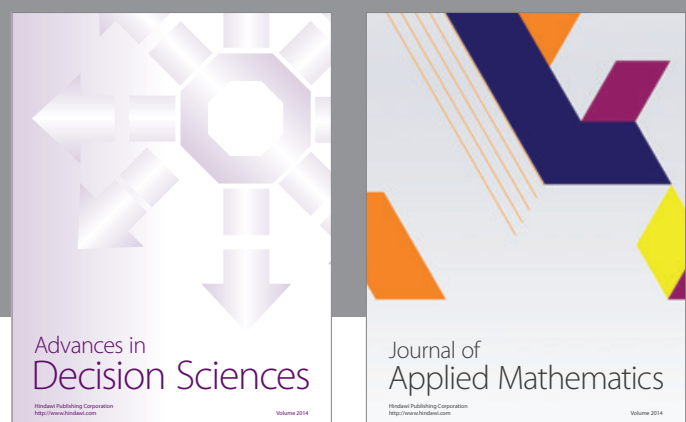

Journal of

Applied Mathematics
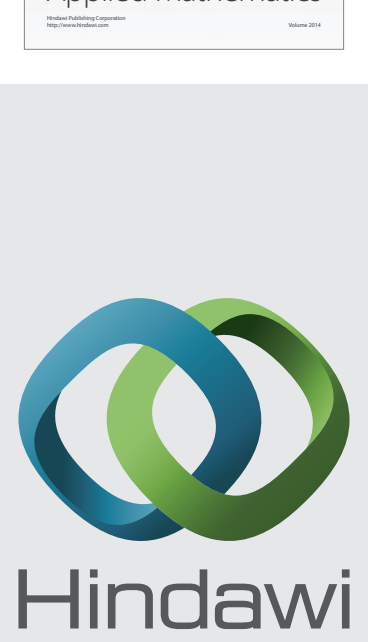

Submit your manuscripts at http://www.hindawi.com
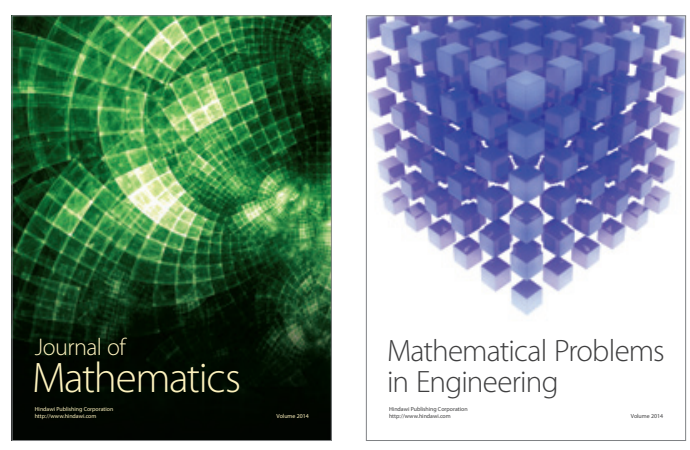

Mathematical Problems in Engineering
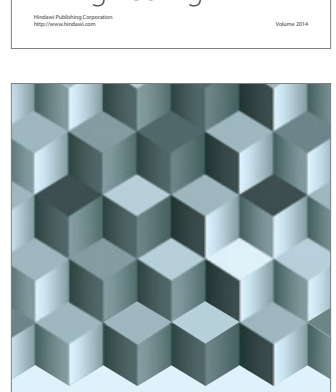

Journal of

Function Spaces
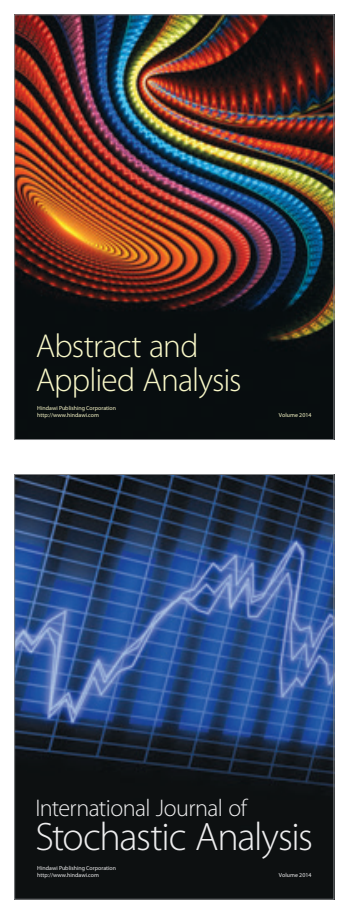

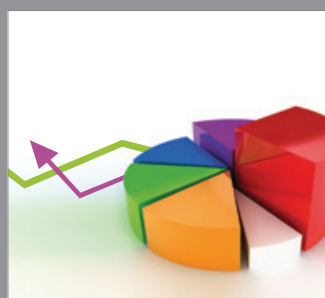

ournal of

Probability and Statistics

Promensencen
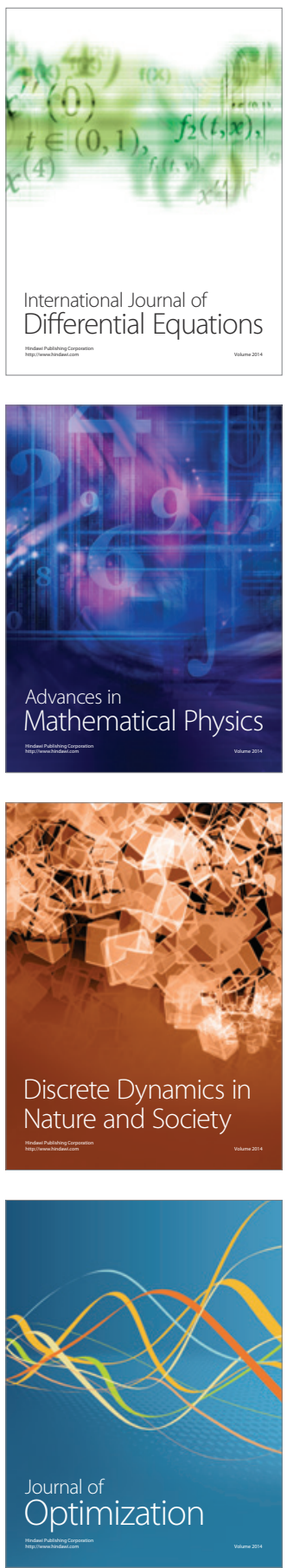